\title{
Genetic Evolution Approach for Target Movement Prediction
}

\author{
Sung Baik ${ }^{1}$, Jerzy Bala ${ }^{2}$, Ali Hadjarian ${ }^{3}$, and Peter Pachowicz ${ }^{3}$ \\ ${ }^{1}$ School of Computer Engineering \\ Sejong University, Seoul 143-747, KOREA \\ sbaik@sejong.ac.kr \\ ${ }^{2}$ School of Information Technology and Engineering \\ George Mason University \\ Fairfax, VA 22030, U.S.A. \\ jbala@gmu.edu \\ ${ }^{3}$ Sigma Systems Research, Inc. \\ Fairfax, VA 22032, U.S.A. \\ \{ahadjarian, ppach\}@sigma-sys.com
}

\begin{abstract}
This paper presents a genetic evolution system, for target movement prediction, which includes functions inferring opponents' strategic movements and displaying such predicted movements in an interactive 3D visualization space. To speed up the analysts' ability to access and integrate information, the prediction approach generates new movements based on past behaviors and application of an inheritance mechanism. It applies Genetic Algorithms (GAs) learning techniques to evolve new individuals in the population of movements in order to converge the evolution process toward optimal movements. The approach is implemented into the GEM (Genetic Evolution of Movement) system and its performance has been experimentally evaluated.
\end{abstract}

\section{Genetic Evolution of Target Movement for Prediction}

GEM uses Genetic Algorithms (GAs) [1-4] to generate the population of movement generation operators. By using crossover and mutation, the population evolves and only the strongest elements survive, thus contributing to improved performance in terms of more probable/optimal movements. This contribution is used as an objective evaluation function to drive the generation process in its search for new and useful movement generation operators. There are 6 steps for GEM's processing (Fig. 1.):

Step 1: Movements Population Seeding. This step sets the initial population of movements. It can use a random selection of initial movements or the Historical Movement Database (HMD). Movements selected from HMD represent historical GMTI target data for scenarios (i.e., tracking and targeting engagements) similar to the one for which the prediction is requested. This step also defines the evaluation function.

Step 2: Binary Encoding. A string representation for each movement is generated using a genetic encoding schema. 
Step 3: Application of Genetic Operators. Crossover and mutation operations are applied during each evolutionary cycle generating additional movements (offspring movements).

Step 4: Binary Decoding. This step executes a reverse process to the binary encoding step (i.e., Step 2 of the algorithm).

Step 5: Evaluation. New movements in the population are evaluated according to the evaluation function defined in Step 1.

Step 6: Recombination. In this step worse performing movements (according to the evaluation function) are discarded from the population leaving the same number of encoded movements in the population as initially set up in Step 1. Step 6 feeds back to Step 2 and restarts the next iteration of the movement generation process.

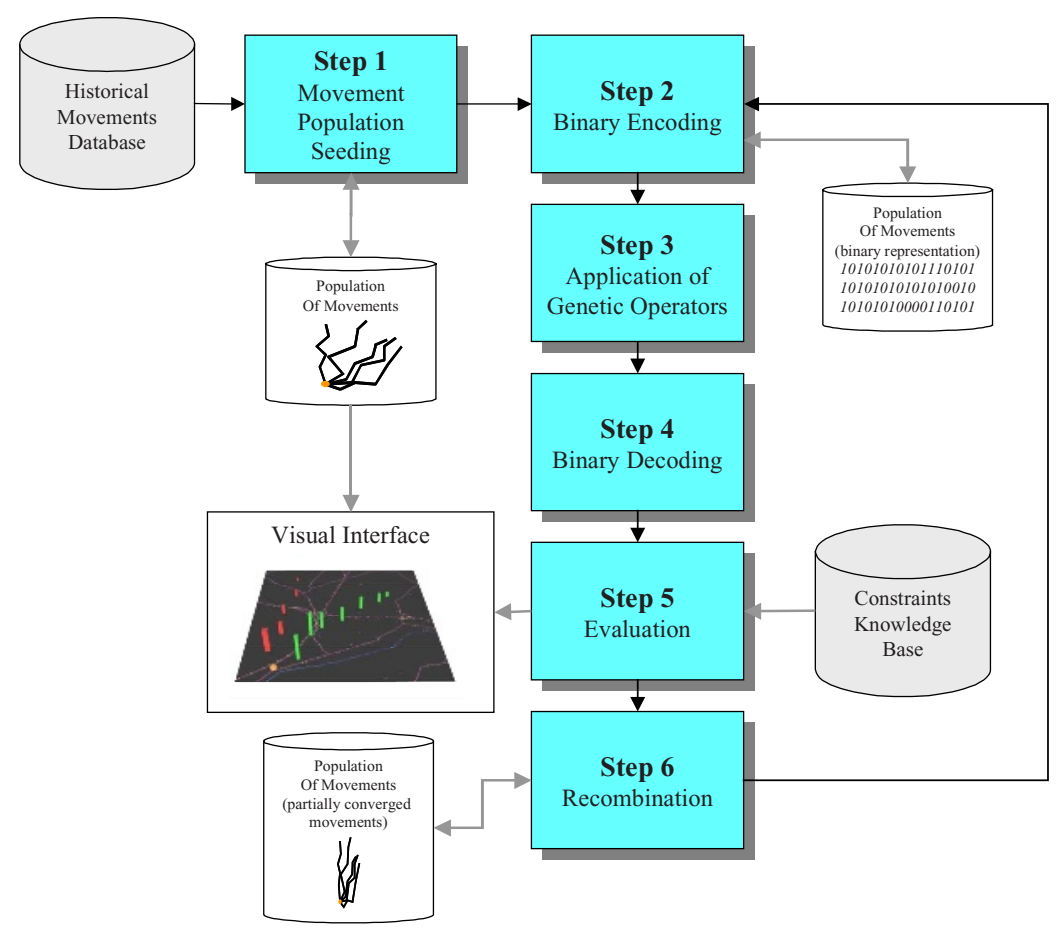

Fig. 1. Movement generation steps

\subsection{GA Operations and Evaluation Function}

The crossover operation, when applied to two operators produces offspring strings (operators). Offspring operators inherit movement characteristics from both their parents and the information about the relevant characteristics can be easily transferred to subsequent generations. This mechanism is possible because the data structure used to represent operators is "linearized." Linearized data structures are those string representations for which the relevance of genetic information is decreasing as one moves from the left towards the right fields/bits (moving through movement timeframes). As 
a consequence, the last operation applied to the current movement causes only minor changes to the operator structure.

The mathematical analysis of GAs shows that they work best when the internal representation encourages the emergence of useful building blocks that can be subsequently recombine to produce improved performance. GEM's string decoding/encoding method identifies these building blocks as the movement fields and subfields in the string representation.

Mutation provides for occasional disturbances in the movement structure to insure diversity in the genetic individuals over long periods of time and to prevent stagnation in the search process for better movements.

The step 5 of GEM's processing evaluates movements in the population according to the evaluation function defined in Step 1. The function scores each movement by calculating partial scores for all $<\mathrm{MSP}, \mathrm{MP}>$ pairs. The partial score is computed by linking $<$ MSP, MP> pair's constraint parameters to the "constraint map". The map consists of several spatially arranged layers representing sets of constraints (e.g., geographical, tactical, communicational, and doctrinal). The location constraint on a given layer represents a probability distribution for the $<\mathrm{MSP}, \mathrm{MP}>$ pair to originate at this location (i.e., MSP to be at this location at timeframe T, and with MP linking this location to the next MSP at timeframe T+1).

Each movement in the genetic population has to be evaluated according to an evaluation function. Each MSP probability at timeframe $\mathrm{T}$ is computed as the product of the location probability value at the $(\mathrm{X}, \mathrm{Y})$ location and the MSP probability at timeframe T-1.

\subsection{Genetic Algorithms}

GAs are iterative procedures which maintain a "population" of candidate solutions $P(t)$ to the objective function $f(x)$ :

$$
P(t)=<x 1(t), x 2(t), \ldots, x N(t)>
$$

Each structure $x i$ in population $P$ is simply a binary string of length L. Generally, each $x i$ represents a vector of parameters to the function $f(x)$, but the semantics associated with the vector is unknown to the GA. During each iteration step, called a "generation", the current population is evaluated, and, on the basis of that evaluation, a new population of candidate solutions is formed.

The initial population $P(0)$ is usually chosen at random. Alternately, the initial population may contain heuristically chosen initial points. In either case, the initial population should contain a wide variety of structures. Each structure in $P(0)$ is then evaluated. For example, if we are trying to minimize a function $\mathrm{f}$, evaluation might consist of computing and storing $f(x l), \ldots, f(x N)$.

The structures of the population $P(t+1)$ are chosen from the population $P(t)$ by a randomized "selection procedure" that ensures that the expected number of times a structure is chosen is proportional to that structure's performance, relative to the rest of the population. That is, if $x j$ has twice the average performance of all the structures in $P(t)$, then $x j$ is expected to appear twice in population $P(t+1)$. At the end of the selection procedure, population $P(t+1)$ contains exact duplicates of the selected structures in population $P(t)$. 
In order to search other points in the search space, some variation is introduced into the new population by means of idealized "genetic recombination operators." The most important recombination operator is called "crossover." Under the crossover operator, two structures in the new population exchange portions of their binary representation. This can be implemented by choosing a point at random, called the crossover point, and exchanging the segments to the right of this point. Termination may be triggered by finding an acceptable approximate solution to $f(x)$, by fixing the total number of evaluations, or some other application dependent criterion.

\section{System Interfacing and Experiments}

A 3D visualization space is used in the GEM system to interface with the user. This interfacing space renders depiction of current movements in the evolved population of movements. It is also used as an editor. The editor can access the Historical Movement Database (HMB) to select and visualize the initial population of movements, to be used for population seeding, visualize intermittent results of the prediction process, and visually control various systems' parameters. This system-interfacing component has been implemented using In3D - a cross-platform Java class library designed to enable the creation of interactive $3 \mathrm{D}$ visualizations of complex information spaces (developed by Visual Insights Corp.)

The performance of the GEM system has been experimentally evaluated on its ability to converge the population of movements to the most probable movement in the lowest number of genetic cycles. After some evolutionary runs of the genetic engine, all movements and their corresponding significant points are mostly converged to one movement. During experiments, a non-convergence result was observed when the evaluation function is formed for a highly multi-modal initial search space. The population of individual movements converged to two separate movements. In such case, the search process has to start from a larger set of initial movements (i.e., search from a larger number of points in the initial cycle of GAs). A higher mutation rate can also potentially alleviate this problem.

\section{References}

1. Bethke, A. D., "Genetic algorithms as function optimizers," Ph.D. Thesis, Dept. Computer and Communication Sciences, Univ. of Michigan, 1981.

2. Brindle, A., "Genetic algorithms for function optimization," Ph.D. Thesis, Computer Science Dept., Univ. of Alberta, 1981.

3. DeJong, K. A., "Adaptive system design: a genetic approach," IEEE Trans. Syst., Man, and Cyber., vol. SMC-10, no. 9, pp. 566-574, Sept. 1980.

4. DeJong, K. A., "Analysis of the behavior of a class of genetic adaptive systems," Ph. D. Thesis, Dept. Computer and Communication Sciences, Univ. of Michigan, 1975. 